POS PROCEEDINGS

\title{
Describing many-particle QM as well as QFT in terms of "single particle" QM with one extra dimension
}

\author{
Roman Sverdlov* \\ Indian Institute of Science Education and Research \\ E-mail: romansverdlov@iisermohali.ac.in
}

From ontological point of view, single particle QM makes a lot more sense than multiparticle case. After all, in case of a single particle you can claim that $\psi$ is NOT "probability amplitude" but, rather, it is simply "classical field"; it simply HAPPENS that probability of collapse of $\psi$ coincides with the square of its absolute value. On the other hand, in case of many particles you can not make this argument: after all, classical field lives in ordinary space, NOT in configuration space! Motivated by this, I am attempting to reduce multiparticle quantum mechanics to single particle one. I am doing this by the following trick. First, I introduce extra dimension. Then I design various "classical" objects in such a way that we have different "classical" configurations on different $x^{5}=$ const hyperplane. Finally, we introduce one SINGLE quantum particle, the one referred to as "pointer", and the "pointer" takes the classical configuration from the hyperplane it resides on and makes it "come true". Thus, instead of quantizing configurations, all we have to do is quantize the location of a pointer, which reduces multiparticle QM to single particle QM. This approach is also extended to reducing quantum field theory to single particle quantum mechanics, as well; we simply replace "classical particle distribution" on the hyperplanes with "field distributions". The reference to the paper where I have done the above work is [1].

References

[1] arXiv:1309.3287

Frontiers of Fundamental Physics 14 - FFP14,

15-18 July 2014

Aix Marseille University (AMU) Saint-Charles Campus, Marseille

${ }^{*}$ Speaker. 